\title{
Galaxies in present-day clusters: evolutionary constraints from their distributions and kinematics
}

\author{
Andrea Biviano ${ }^{1}$ and Peter Katgert ${ }^{2}$ \\ ${ }^{1}$ INAF/Osservatorio Astronomico di Trieste, via G. B. Tiepolo 11, I-34131 Trieste, Italy \\ ${ }^{2}$ Sterrewacht Leiden, Postbus 9513, Niels Bohrweg 2, 2300 RA Leiden, The Netherlands
}

\begin{abstract}
We discuss evidence in local, present-day clusters of galaxies (from the ENACS survey) about the way in which those clusters have evolved and about the evolutionary relationships between the galaxies of different morphological types in them. This evidence is complementary to that obtained from the study of clusters at intermediate and high redshifts. We argue that the spatial distribution and the kinematics of the various types of galaxies in and outside substructures support the following picture.

The elliptical and SO galaxies have been around for a long time and have obtained an isotropic velocity distribution. The spatial distribution and kinematics of the early spirals are consistent with the idea that many of their kind have transformed into an S0, but that they have survived, most likely because of their velocities. The distribution and kinematics of the late spirals are consistent with a picture in which they have been accreted fairly recently. They have mildly radial orbits and hardly populate the central regions, most likely because they suffer tidal disruption. Finally, the distribution and kinematics of the galaxies in substructures, when taken at face value, imply tangential velocity anisotropy for these galaxies, but this result may be (partly) due to the procedure by which these galaxies are selected. A first attempt to take the effects of selection into account shows that isotropic (or even mildly radial) orbits of subcluster galaxies cannot be excluded.
\end{abstract}

\section{Introduction and summary of the data sample}

By observing the precursors of present-day clusters, it has become possible to study in a direct manner the evolution of the clusters themselves, and the evolutionary relationships between the various types of galaxies in them. Images of rich clusters at redshifts $z \approx 1$ show a high merger fraction, which is taken to be direct evidence for the formation of elliptical galaxies by merging. Yet, it is unlikely that all early-type galaxies formed around or before that epoch (van Dokkum \& Franx 2001). In particular, the variation with redshift of the composition of the galaxy population in clusters suggests that S0 galaxies have come into being fairly recently, say, since $z \sim 0.5$, apparently at the expense of the spirals (Dressler et al. 1997; Fasano et al. 2000). The processes by which spirals transform into S0 galaxies have been studied numerically (e.g. Moore et al. 1998; Abadi et al. 1999). It appears that impulsive encounters can indeed transform a spiral into an S0 galaxy, by stripping a small fraction of the stellar disk, and by heating up the disk.

We have used the data from the ENACS to investigate if the effects of these processes are still detectable in present-day clusters. Our analysis is based on an 'ensemble' cluster of $\sim 3000$ galaxies built by combining 59 nearby $(0.035 \leqslant z \leqslant 0.098)$ clusters from the ENACS data-set (Katgert et al. 1996, 1998), after suitable scaling of the galaxies projected clustercentric distances and velocities (Biviano et al. 2002). For most of these galaxies also spectral-type or morphological data are available (Thomas 2002). We consider five populations of cluster galaxies, selected on the basis of their different projected 
phase-space distributions (Biviano et al. 2002). These populations are (i) galaxies in subclusters (herafter referred to as the 'Subs' class), and, among the galaxies outside subclusters, (ii) the brightest ellipticals (with $M_{R} \leqslant-22+5 \log h$, the 'Bright' class), (iii) the other ellipticals together with the E/S0 and the S0 galaxies (the 'Early' class), (iv) the early spirals ( $\mathrm{Sa}-\mathrm{Sb}$; the 'S-early' class), and (v) the late spirals and irregulars (Sbc-Ir) together with the emission-line galaxies (except those with early morphology), hereafter globally referred to as the 'S-late' class galaxies.

The substructure distinction is based on a refined version of the Dressler \& Shectman (1988) criterion. Galaxies in cold and/or moving substructures were identified by the velocity dispersion and/or average velocity of their surroundings, which are smaller than the global value, or different from the cluster mean, respectively. With the significance threshold that we used, about $25 \%$ of all galaxies are in substructures. The fraction of galaxies in substructures shows a significant apparent decrease towards the cluster center. This is probably a real effect, but there is likely to be a systematic contribution from the selection algorithm (Biviano et al. 2002).

\section{Clues from the spatial distributions}

The projected distributions of the 5 classes are shown in the left-hand panel of Fig. 1, in the form of the smooth estimate of $I(R)$ (using the LOWESS technique, see Gebhardt \& Fisher 1995; Katgert et al. 2004; Biviano \& Katgert 2004). Note that our cluster sample has a median value $r_{200} \approx 1.2 h^{-1} \mathrm{Mpc}$. The brightest ellipticals (Bright-class galaxies) are most centrally concentrated. The Early-class shows a flatter slope, however with a clear steepening beyond $\approx 0.3 r_{200}$. The S-early- and S-late- classes both avoid the central region within $\approx 0.1 r_{200}$, but in addition the S-late-class shows an appreciable flattening of $I(R)$ below $\approx 0.3 r_{200}$. The Abel deprojection of $I(R)$ which yields the 3-D number density profiles shows that both the S-early- and S-late- classes are absent from the central region. Apparently, galaxies of the S-early- and S-late- classes get destroyed or transformed within $\approx 0.3 r_{200}$, as it is unlikely that their phase-space distributions would prohibit them from entering the central regions. Galaxies in substructures do not avoid the central regions, but are less centrally concentrated than galaxies of the Early-class.

Information on possible transformation relations between the galaxies in the different classes may be gleaned from the morphology-radius (MR) and, in particular, the morphology-density (MD) relation (e.g. Dressler 1980). For the ENACS clusters those have been discussed by Thomas \& Katgert (2004), who considered ellipticals (including the brightest) and S0 galaxies separately, in addition to the S-early- and S-late-classes. The MR-relations show the dependence on radius of the composition in terms of morphology, and are fully consistent with the $I(R)$ 's in the upper panel of Fig. 1. The MD-relation gives the distribution of local projected density around galaxies of the different classes. Thomas \& Katgert (2004) first used the original definition of projected density, i.e. that based on the 10 nearest neigbours, or $\Sigma_{10}$. However, $\Sigma_{10}$ contains a very noticeable print-through of the average relation between projected density and radius. As a result, the $\Sigma_{10}$ MD-relation is highly correlated with the MR-relation (and thus, with the $I(R)^{\prime} s$ ). The ellipticals prefer the highest densities, and the remaining three classes have indistinguishable $\Sigma_{10}$ distributions shifted towards lower densities (left-hand panel of Fig. 2). From this perspective, the S0 galaxies and ellipticals behave differently; part of this difference is due to the brightest ellipticals being located at the clusters centres.

Thomas \& Katgert (2004) also used $\Sigma_{1}$, i.e. the projected density based on the distance to the nearest neighbour. As expected, $\Sigma_{1}$ is much noisier than $\Sigma_{10}$, but at the same time 


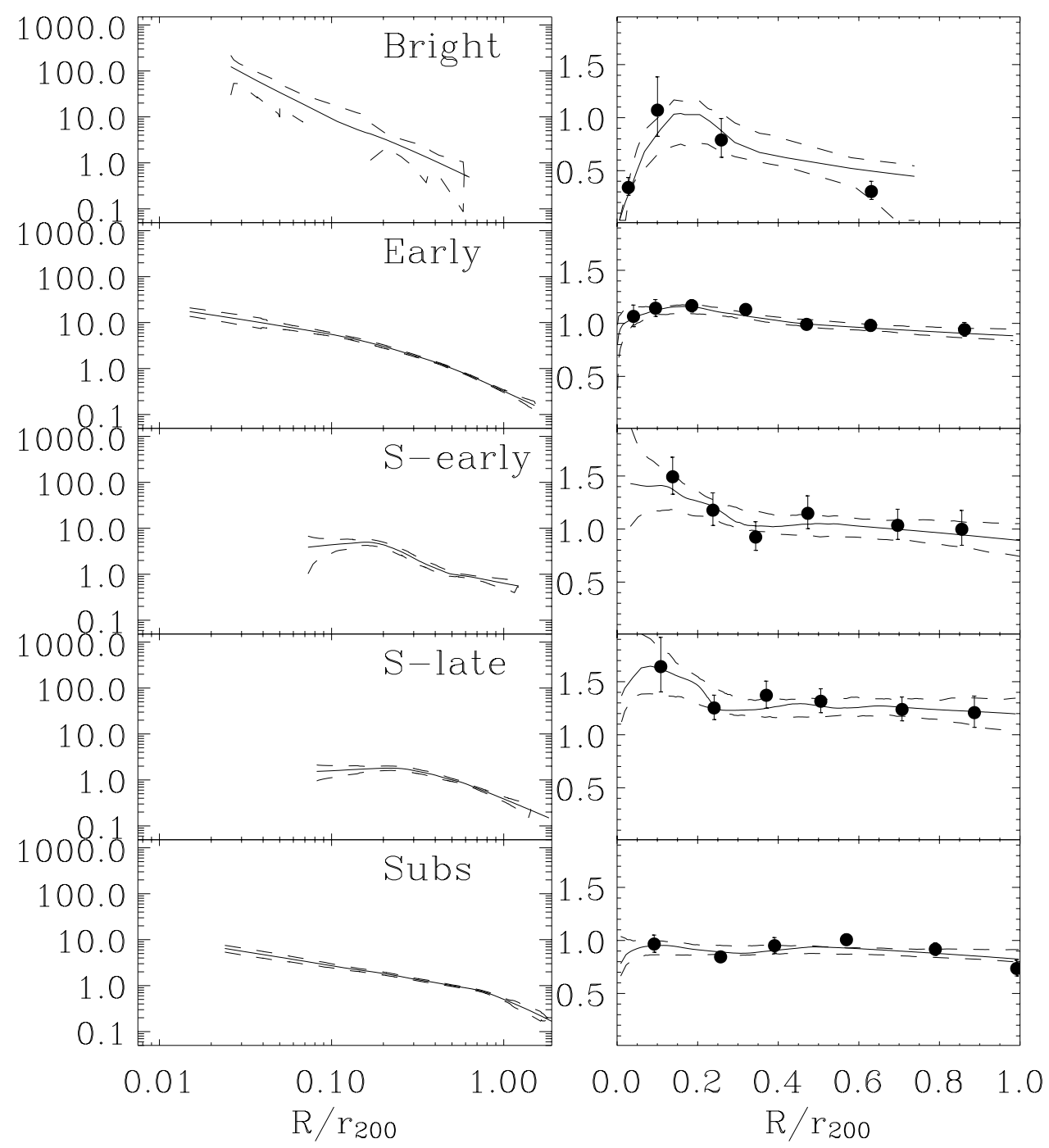

Figure 1. The projected distributions (left) and the velocity dispersion profiles (right) of the five galaxy classes. Dashed lines indicate 1- $\sigma$ confidence levels. Dots (with 1- $\sigma$ error bars) in the right-hand panel indicate binned estimates of the velocity dispersion profiles. Units along the $\mathrm{y}$-axis in the left-hand panel are arbitrary. The y-axis in the right-hand panel is in units of the cluster global line-of-sight velocity dispersion (on average, $\approx 700 \mathrm{~km} \mathrm{~s}^{-1}$ for the 59 clusters that compose the ensemble cluster).

it is much less correlated with projected radius, and therefore a less biased measure of local projected density than $\Sigma_{10}$. That is probably the reason why the $\Sigma_{1}$-distributions of S0 and S-early galaxies are significantly different, with S0 galaxies preferring higher densities than the S-early galaxies (right-hand panel of Fig. 2). 

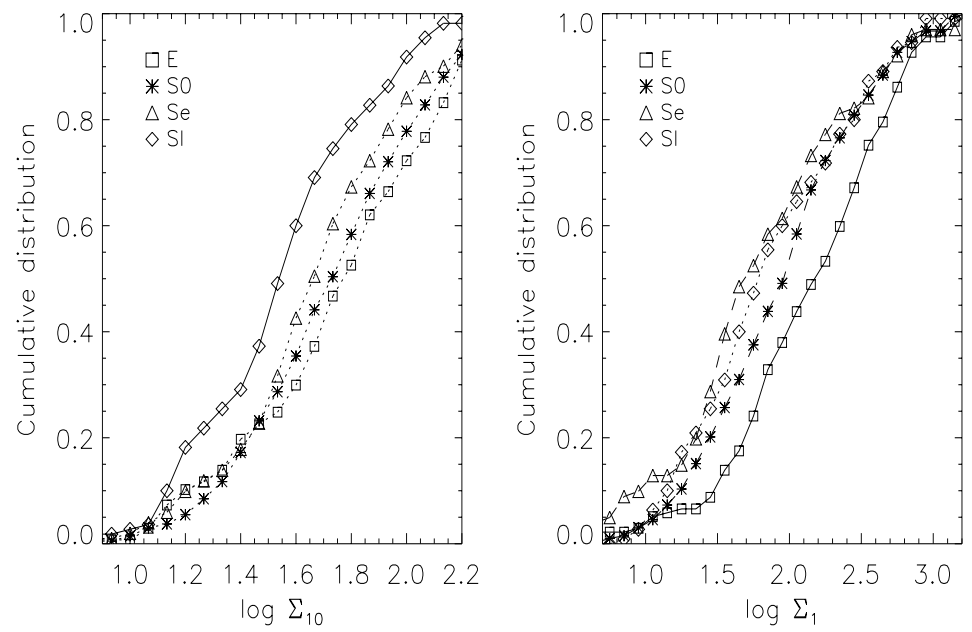

Figure 2. The morphology-density relations for $\Sigma_{10}$ (left) and $\Sigma_{1}$ (right).

\section{Clues from the kinematics}

\subsection{Isotropic orbit distributions?}

For a study of the kinematics of the various galaxy classes, we have used the observed projected distributions $I(R)$ and velocity dispersion profiles (vdp's, hereafter). The vdp's of the five different classes are quite different (see right-hand panel of Fig. 1). The Brightclass vdp is quite 'cold', not only in the cluster centre. The Early-class vdp is nearly flat. The vdp's of the S-early and the S-late classes are rather similar, showing a steep decline from the centre out to $\sim 0.3 r_{200}$, followed by a nearly flat vdp. However, the S-late vdp is 'hotter' than the S-early vdp at all radii. Finally, the vdp of the Subs-class is cold and flat, even flatter than the Early-class vdp.

In order to determine the kinematics of the different galaxy classes we first need to determine the gravitational potential in which the galaxies move. We have therefore first derived the mass-profile $M(<r)$, using the data for the Early-class galaxies, assuming that those have an isotropic distribution of orbits. That assumption is justified by the shape of their line-of-sight velocity distribution, from which Katgert et al. (2004) concluded that, for the Early-class $0.8 \leqslant \beta^{\prime} \leqslant 1.05$, with

$$
\beta^{\prime} \equiv\left(\overline{v_{r}^{2}} / \overline{v_{t}^{2}}\right)^{1 / 2}
$$

where $\overline{v_{r}^{2}}, \overline{v_{t}^{2}}$ are the mean squared components of the radial and tangential velocity, respectively (i.e. isotropic orbits correspond to $\beta^{\prime}(r)=1$ ). Therefore the cluster mass profile, $M(<r)$, follows from the isotropic Jeans equation (Binney \& Tremaine 1987) applied to the Early-class. The resulting $M(<r)$ is rather similar to a NFW (Navarro et al. 1997) profile with a concentration parameter $c=4.0_{-1.5}^{+2.7}$ (Katgert et al. 2004).

Next, this cluster $M(<r)$ was used to search for solutions of dynamical equilibrium for the other four classes (see $\S 1$ ). Given the cluster $M(<r)$ and the observed number density profile of a given class of cluster galaxies, one can solve the inverse Jeans equation (van der Marel 1994) for a given $\beta^{\prime}(r)$. The resulting vdp is then compared, after the usual Abel projection (Binney \& Tremaine 1987), with the observed line-of-sight vdp, in a 
$\chi^{2}$ sense. If the two vdp's are statistically indistinguishable, the $\beta^{\prime}(r)$ profile is considered acceptable. Instead of considering complicated $\beta^{\prime}(r)$ profiles, we tested whether the data were consistent with an isotropic orbit distribution for the other galaxy classes, i.e. if $\beta^{\prime}(r)=1$ is acceptable. This appears to be the case for galaxies of the S-early class, but not for those of the Bright, S-late, and Subs classes.

\subsection{The orbital anisotropy profiles}

Since the assumption of (nearly) isotropic orbits is not valid for all except the Early and, possibly, the S-early classes we have investigated what the data tell us about the orbital anisotropy. Instead of trying constant anisotropy (van der Marel et al. 2000; Lokas \& Mamon 2003), or anisotropy models (Carlberg et al. 1997), we determine $\beta^{\prime}(r)$ directly from the data, using the procedure that Solanes \& Salvador-Solé (1990) developed from that of Binney \& Mamon (1982). The $\beta^{\prime}(r)$ solutions are checked by inverting the Jeans equation again (van der Marel 1994), thereby deriving a vdp that can be compared with the observed one. The derived vdp's always agree very well with the observed vdp's. We also applied this check to the sample of Early-class galaxies, and indeed recovered $\beta^{\prime}(r)=1$ to within $\pm 15 \%$.

Uncertainties on the $\beta^{\prime}$-profiles were estimated by dividing each galaxy sample into four subsamples, each containing a different half of all the galaxies in the original sample. We then applied the procedure of Solanes \& Salvador-Solé (1990) to each subsample. The rms of the four resulting $\beta^{\prime}$-profiles is an approximate, but probably conservative, estimate of the uncertainty in the full sample $\beta^{\prime}(r)$.

Physically acceptable solutions are found for the Early and three of the four other populations of cluster galaxies. No acceptable solution is found for the Bright class. This is most likely the consequence of a failure of the collisionless Jeans equation in describing the dynamics of these galaxies, which is probably affected by dissipative processes, such as mergers and dynamical friction (see Fusco-Femiano \& Menci 1998 for a collisional solution).

The $\beta^{\prime}$-profile obtained for the S-early class increases rapidly from $\beta^{\prime} \simeq 1$ near the centre, to $\beta^{\prime} \simeq 1.8$ at $r / r_{200} \simeq 0.45$, and then smoothly decreases to $\simeq 1$ again at larger radii (see Fig. 3, top panel). Taken at face value, this solution implies mild radial anisotropy just outside the cluster centre. The partial radial anisotropy at $r \simeq 0.45 r_{200}$ could result from a natural selection effect. I.e., among S-early galaxies, those reaching the high-density inner cluster region can avoid transforming into S0's only if they move sufficiently fast. We should not overinterpret the data, though, since the isotropic solution is acceptable for galaxies of the S-early class, and the $\beta^{\prime}$-profile is in fact consistent with $\beta^{\prime}(r)=1$.

The $\beta^{\prime}(r)$ profile of the S-late galaxies is not different from unity out to $\simeq 0.7 r_{200}$, but then increases linearly with radius reaching a radial anisotropy $\beta^{\prime} \simeq 1.8$ at $r \simeq$ $1.5 r_{200}$ (see Fig. 3, mid panel). This $\beta^{\prime}$-profile is remarkably similar to those obtained for dark matter particles in numerical simulations (Ghigna et al. 1998; Diaferio 1999). It is therefore tempting to conclude, by analogy, that S-late galaxies, like dark matter particles in numerical simulations, still retain memory of the process of (mostly radial) gravitational infall along the filaments connecting to the cluster, and are therefore recent arrivals into the cluster.

Finally, the data for the galaxies of the Subs class imply orbits with substantial tangential anisotropy at all radii (see Fig. 3, solid line in the bottom panel). However, two systematic effect must be accounted for. The first one is the internal velocity dispersion of subclusters. If we subtract an assumed internal velocity dispersion of $250 \mathrm{~km} \mathrm{~s}^{-1}$ from the observed vdp of Subs galaxies, the orbital tangential anisotropy becomes even 


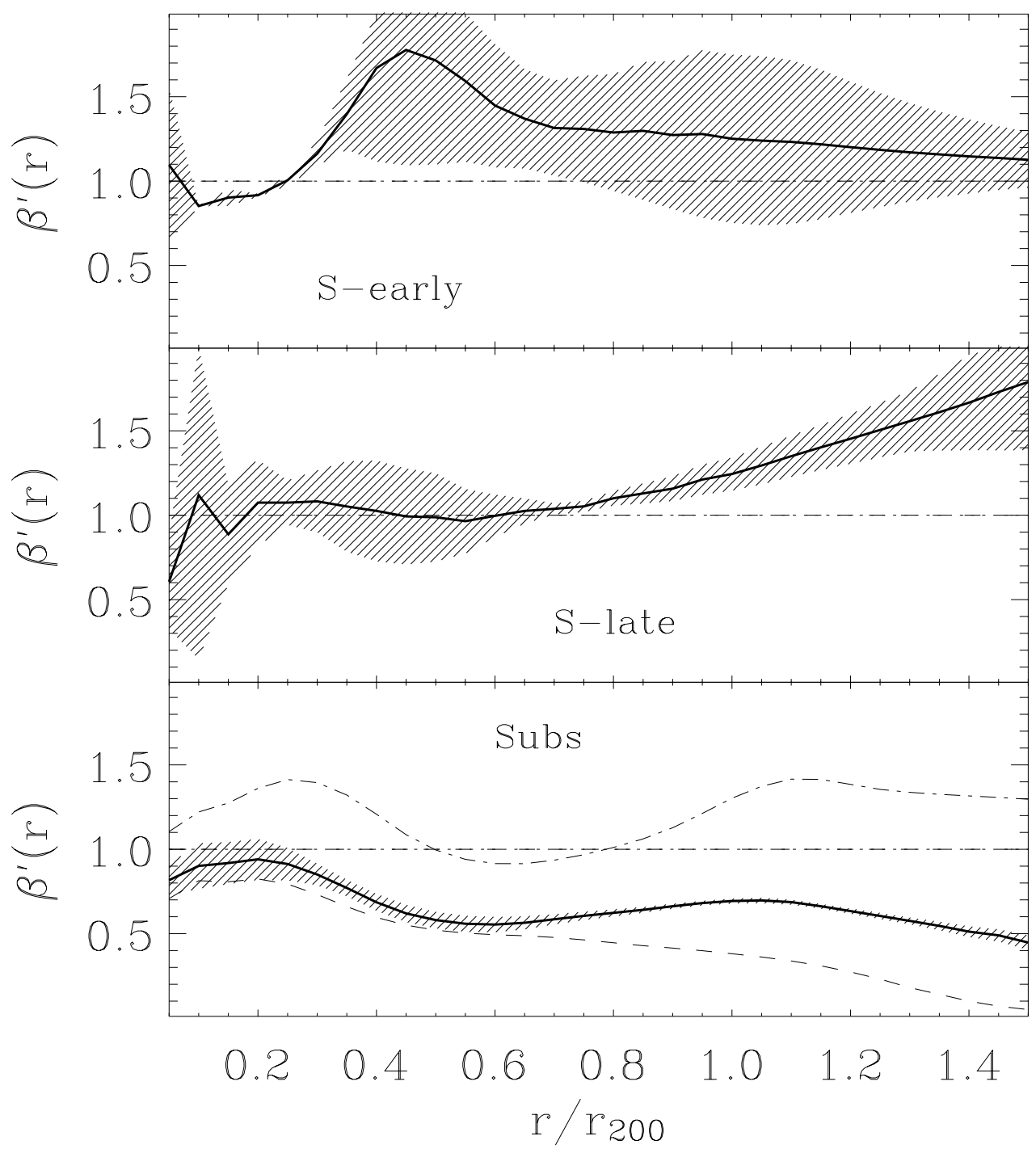

Figure 3. Top panel: The velocity anisotropy profile, $\beta^{\prime}(r) \equiv\left(\overline{v_{r}^{2}}(r) / \overline{v_{t}^{2}}(r)\right)^{1 / 2}$, of the S-early class. The hatched region represents the approximate 1- $\sigma$ confidence interval. Mid panel: same as top panel, but for the S-late class. Bottom panel: same as top panel, but for the Subs class. The dashed line is the $\beta^{\prime}$-profile obtained after subtracting in quadrature the internal velocity dispersion of the subclusters from the observed vdp. The dash-dotted line is the $\beta^{\prime}$-profile obtained after correcting the observed number density profile and vdp of the Subs class for the systematic selection effects, according to a plausible, but by no means unique, model.

stronger (see Fig. 3, dashed line in the bottom panel). The second effect is the radial variation of the efficiency of the detection of Subs galaxies. Very simple modeling shows that the efficiency of the selection method decreases towards the cluster center. As a result, both the number density profile and the vdp of the Subs class are affected. A simple first-order correction of these profiles leads to a $\beta^{\prime}$-profile which no longer implies tangential orbits (see Fig. 3, dash-dotted line in the bottom panel). A better understanding of the orbital characteristics of subclusters thus requires either an improved, 
radially-unbiased, selection method of galaxies in subclusters, or at least an improved modeling of the biases.

\section{Summary}

The Early-class galaxies (ellipticals and S0 galaxies) in clusters have a distribution of line-of-sight velocities that is consistent with an isotropic orbit distribution. These galaxies were therefore used to derive the mass profile of the ENACS ensemble cluster. Using this mass profile, we constrained the orbits of cluster galaxies of other classes. From the distribution and velocity dispersion profile of the S-early-class we conclude that these may also have isotropic orbits, although they show an apparent radial anisotropy around $\approx 0.45 r_{200}$. The latter may be a result of the process by which the majority of the S-earlygalaxies are thought to have transformed into S0 galaxies through impulsive encounters. The fact that the local density around S-early-galaxies is smaller than that around S0 galaxies provides further support for this interpretation. The S-late-class has mildly radial orbits outside $\approx 0.7 r_{200}$, and are probably still falling in. Their absence from the central region most likely indicates that they are tidally disrupted on their crossing of the cluster core. Finally, the galaxies in substructures apparently are on tangential orbits, although isotropic (or mildly radial) orbits cannot be excluded at present.

\section{Acknowledgements}

We wish to thank the Local Organizing Committee for a very enjoyable and interesting meeting. We acknowledge useful discussions with Alain Mazure and Tom Thomas.

\section{References}

Abadi, M. G., Moore, B., \& Bower, R., G. 1999, MNRAS, 308, 947.

Binney, J., \& Mamon, G. 1982, MNRAS, 200, 361.

Binney, J. \& Tremaine, S. 1987, "Galactic Dynamics" (Princeton Univ. Press).

Biviano, A., Katgert, P., Thomas, T., \& Adami, C. 2002, A\& $A$, 387, 8.

Biviano, A., \& Katgert, P. 2004, in preparation.

Carlberg, R.G., Yee, H.K.C., \& Ellingson, E. 1997, ApJ, 478, 462.

Diaferio, A. 1999, MNRAS, 309, 610.

Dressler, A. 1980, ApJ, 236, 351.

Dressler, A. \& Shectman, S.A. 1988, AJ, 95, 985.

Dressler, A., Oemler, A. Jr., Couch, W. J., et al. 1997, ApJ, 490, 577.

Fasano, G., Poggianti, B. M., Couch, W. J., et al. 2000, ApJ, 542, 673.

Fusco-Femiano, R. \& Menci, N. 1998, ApJ, 498, 95.

Gebhardt, K. \& Fischer, P. 1995, AJ, 109, 209

Ghigna, S., Moore, B., Governato, F., et al. 1998, MNRAS, 300, 146.

Katgert, P., Mazure, A., Perea, J., et al. 1996, $A \mathscr{\xi} A$, 310, 8.

Katgert, P., Mazure, A., den Hartog, R., et al. 1998, A\&AS, 129, 399.

Katgert, P., Biviano, A., \& Mazure, A. 2004, ApJ, 600, 657.

Lokas, E. \& Mamon, G. 2003, MNRAS, 343, 401.

Moore, B., Lake, G., \& Katz, N. 1998, ApJ, 495, 139.

Navarro, J.F., Frenk, C.S. \& White, S.D.M. 1997, ApJ, 490, 493.

Solanes, J.M., \& Salvador-Solé 1990, A\& A, 234, 93.

Thomas, T. 2002, PhD thesis, Leiden Observatory.

Thomas, T., \& Katgert, P. 2004, in preparation.

van der Marel, R. 1994, MNRAS, 270, 271.

van der Marel, R., Magorrian, J., Carlberg, R., et al. 2000, AJ, 119, 2038.

van Dokkum, P. G., \& Franx, M. 2001, ApJ, 553, 90. 